\title{
Correction to: Impact of cGVHD on socioeconomic outcomes in survivors with pediatric hematopoietic stem cell transplant in Japan: a cross-sectional observational study
}

Takafumi Soejima $^{1,2}$ (D) Masaaki Shiohara ${ }^{3} \cdot$ Yasushi Ishida $^{4} \cdot$ Masami Inoue $^{5} \cdot$ Akira Hayakawa $^{6} \cdot$ Atsushi Sato $^{7}$. Kiyoko Kamibeppu ${ }^{1,2} \cdot$ Yoshiko Atsuta $^{8,9} \cdot$ Takuya Yamashita $^{10}$

Published online: 12 February 2021

(c) Japanese Society of Hematology 2021

\section{Correction to: International Journal of Hematology https://doi.org/10.1007/s12185-020-03058-7}

In the original publication of the article, the Tables 1 and 2 were published incorrectly. The correct Tables 1 and 2 are given in this correction.

The original article can be found online at https://doi.org/10.1007/ s12185-020-03058-7.

Takafumi Soejima

tsoeji-tky@umin.ac.jp

1 Department of Family Nursing, School of Health Sciences and Nursing, Graduate School of Medicine, The University of Tokyo, 7-3-1, Hongo, Bunkyo-ku, Tokyo 113-0033, Japan

2 Department of Health Quality and Outcome Research, Division of Nursing Systems, Global Nursing Research Center, Graduate School of Medicine, The University of Tokyo, Tokyo, Japan

3 Department of Pediatrics, Matsumoto Dental University Hospital, Shiojiri, Japan

4 Pediatric Medical Center, Ehime Prefectural Central Hospital, Matsuyama, Japan
5 Department of Hematology/Oncology, Osaka Women's and Children's Hospital, Osaka, Japan

6 Department of Palliative Care, Yodogawa Christian Hospital, Osaka, Japan

7 Department of Hematology/Oncology, Miyagi Children's Hospital, Sendai, Japan

8 Japanese Data Center for Hematopoietic Cell Transplantation, Nagoya, Japan

9 Department of Healthcare Administration, Nagoya University Graduate School of Medicine, Nagoya, Japan

10 Department of Hematology/Oncology, St. Luke's International Hospital, Tokyo, Japan 
Table 1 Demographic and clinical characteristics in HCT survivors aged $8-15$ years

\begin{tabular}{|c|c|c|c|c|c|}
\hline & \multicolumn{2}{|c|}{ Auto-HCT $(n=38)$} & \multicolumn{2}{|c|}{ Allo-HCT $(n=150)$} & \multirow[b]{2}{*}{$p^{\mathrm{a}}$} \\
\hline & Median & IQR & Median & IQR & \\
\hline Age at the time of the survey (year) & 11.0 & $9.0-13.0$ & 12.0 & $10.0-14.0$ & 0.023 \\
\hline Age at HCT (year) & 2.5 & $1.0-5.0$ & 4.0 & $2.0-6.0$ & 0.017 \\
\hline \multirow[t]{2}{*}{ Time elapsed since HCT (year) } & 7.7 & $6.2-9.9$ & 7.5 & $5.1-9.5$ & 0.392 \\
\hline & $n$ & $\%$ & $n$ & $\%$ & $p^{\mathrm{b}}$ \\
\hline Female gender & 15 & 40 & 60 & 40 & 1.000 \\
\hline \multicolumn{6}{|l|}{ Diagnosis } \\
\hline ALL & 0 & 0 & 47 & 31 & $<0.001$ \\
\hline AML & 0 & 0 & 25 & 17 & \\
\hline Hematopoietic dysfunction & 0 & 0 & 29 & 19 & \\
\hline Lymphoma & 1 & 3 & 1 & 1 & \\
\hline CML & 0 & 0 & 3 & 2 & \\
\hline Myelodysplastic syndrome & 1 & 3 & 10 & 7 & \\
\hline Hemophagocytic syndrome & 0 & 0 & 7 & 5 & \\
\hline Primary immunodeficiency syndrome & 0 & 0 & 19 & 13 & \\
\hline Congenital metabolic disorder & 0 & 0 & 5 & 3 & \\
\hline Solid tumor & 36 & 95 & 4 & 3 & \\
\hline \multicolumn{6}{|l|}{ Number of transplantation } \\
\hline Once & 35 & 92 & 137 & 91 & 1.000 \\
\hline More than twice & 3 & 8 & 13 & 9 & \\
\hline \multicolumn{6}{|l|}{ Year at HCT } \\
\hline-2000 & 1 & 3 & 5 & 3 & 1.000 \\
\hline $2001-2005$ & 16 & 42 & 63 & 42 & \\
\hline $2006-$ & 21 & 55 & 82 & 55 & \\
\hline \multicolumn{6}{|l|}{ Stem cell source } \\
\hline Bone marrow & 13 & 34 & 99 & 66 & $<0.001$ \\
\hline Peripheral blood & 25 & 66 & 8 & 5 & \\
\hline Cord blood & 0 & 0 & 43 & 29 & \\
\hline \multicolumn{6}{|l|}{ Donor type } \\
\hline Unrelated & - & & 90 & 60 & \\
\hline \multicolumn{6}{|l|}{ Conditioning regimen } \\
\hline Myeloablative & - & & $81 / 108$ & 75 & \\
\hline Busulfan & $0 / 33$ & 0 & $21 / 133$ & 16 & 0.009 \\
\hline Cyclophosphamide & $1 / 33$ & 3 & $97 / 145$ & 67 & $<0.001$ \\
\hline Total body irradiation & $5 / 33$ & 15 & $73 / 139$ & 53 & $<0.001$ \\
\hline \multicolumn{6}{|c|}{ Administration of immunosuppressants for GVHD } \\
\hline Steroid & - & & $14 / 132$ & 11 & \\
\hline Cyclosporine & - & & $46 / 130$ & 35 & \\
\hline Tacrolimus & - & & $94 / 146$ & 64 & \\
\hline Methotrexate & - & & $123 / 145$ & 85 & \\
\hline \multicolumn{6}{|l|}{ cGVHD symptoms } \\
\hline Presence & - & & $63 / 148$ & 43 & \\
\hline Skin & - & & $21 / 148$ & 14 & \\
\hline Oral & - & & $13 / 148$ & 9 & \\
\hline Eye & - & & $7 / 148$ & 5 & \\
\hline Lung & - & & $6 / 148$ & 4 & \\
\hline Gastrointestinal & - & & $3 / 148$ & 2 & \\
\hline Liver & - & & $10 / 148$ & 7 & \\
\hline Joint/muscle & - & & 9/148 & 6 & \\
\hline Genital & - & & $2 / 148$ & 1 & \\
\hline
\end{tabular}

$A L L$ acute lymphoblastic leukemia, $A M L$ acute myeloid leukemia, $c G V H D$ chronic graft-versus-host dis ease, $C M L$ chronic myeloid leukemia, $H C T$ hematopoietic stem cell transplantation, $I Q R$ interquartile range ${ }^{a}$ Mann-Whitney $U$ test

${ }^{\mathrm{b}}$ Fisher's exact test 
Table 2 Demographic and clinical characteristics in HCT survivors aged $\geq 16$ years

\begin{tabular}{|c|c|c|c|c|c|}
\hline & \multicolumn{2}{|c|}{ Auto-HCT $(n=32)$} & \multicolumn{2}{|c|}{ Allo-HCT $(n=222)$} & \multirow[b]{2}{*}{$p^{\mathrm{a}}$} \\
\hline & Median & IQR & Median & IQR & \\
\hline Age at the time of the survey (year) & 20.5 & $18.0-29.0$ & 20.0 & $18.0-24.0$ & 0.330 \\
\hline Age at HCT (year) & 9.0 & $4.0-14.8$ & 10.0 & $7.0-13.0$ & 0.415 \\
\hline \multirow[t]{2}{*}{ Time elapsed since HCT (year) } & 13.8 & $10.9-16.7$ & 10.6 & $7.4-14.5$ & 0.005 \\
\hline & $\mathrm{n}$ & $\%$ & $\mathrm{n}$ & $\%$ & $p^{\mathrm{b}}$ \\
\hline Female gender & 17 & 53 & 115 & 52 & 1.000 \\
\hline \multicolumn{6}{|l|}{ Diagnosis } \\
\hline ALL & 6 & 19 & $76 / 221$ & 34 & $<0.001$ \\
\hline AML & 3 & 9 & $37 / 221$ & 17 & \\
\hline Hematopoietic dysfunction & 0 & 0 & $45 / 221$ & 20 & \\
\hline Lymphoma & 4 & 13 & $13 / 221$ & 6 & \\
\hline CML & 0 & 0 & $14 / 221$ & 6 & \\
\hline Myelodysplastic syndrome & 0 & 0 & $14 / 221$ & 6 & \\
\hline Hemophagocytic syndrome & 0 & 0 & $4 / 221$ & 2 & \\
\hline Primary immunodeficiency syndrome & 0 & 0 & $7 / 221$ & 3 & \\
\hline Congenital metabolic disorder & 0 & 0 & $8 / 221$ & 4 & \\
\hline Solid tumor & 19 & 59 & $3 / 221$ & 1 & \\
\hline \multicolumn{6}{|l|}{ Number of transplantation } \\
\hline Once & 29 & 91 & 201 & 91 & 1.000 \\
\hline More than twice & 3 & 9 & 21 & 10 & \\
\hline \multicolumn{6}{|l|}{ Year at HCT } \\
\hline-2000 & 20 & 63 & 78 & 35 & 0.016 \\
\hline 2001-2005 & 7 & 22 & 80 & 36 & \\
\hline $2006-$ & 5 & 16 & 64 & 29 & \\
\hline \multicolumn{6}{|l|}{ Stem cell source } \\
\hline Bone marrow & 10 & 31 & 179 & 81 & $<0.001$ \\
\hline Peripheral blood & 22 & 69 & 15 & 7 & \\
\hline Cord blood & 0 & 0 & 28 & 13 & \\
\hline \multicolumn{6}{|l|}{ Donor type } \\
\hline Unrelated & - & & 130 & 59 & \\
\hline \multicolumn{6}{|l|}{ Conditioning regimen } \\
\hline Myeloablative & - & & $117 / 151$ & 78 & \\
\hline Busulfan & $8 / 31$ & 26 & $33 / 175$ & 19 & 0.463 \\
\hline Cyclophosphamide & $4 / 31$ & 13 & $133 / 203$ & 66 & $<0.001$ \\
\hline Total body irradiation & $5 / 31$ & 16 & $133 / 194$ & 69 & $<0.001$ \\
\hline \multicolumn{6}{|c|}{ Administration of immunosuppressants for GVHD } \\
\hline Steroid & - & & $14 / 172$ & 8 & \\
\hline Cyclosporine & - & & $89 / 178$ & 50 & \\
\hline Tacrolimus & - & & $112 / 204$ & 55 & \\
\hline Methotrexate & - & & $181 / 208$ & 87 & \\
\hline \multicolumn{6}{|l|}{ cGVHD symptoms } \\
\hline Presence & - & & $105 / 222$ & 47 & \\
\hline Skin & - & & $39 / 222$ & 18 & \\
\hline Oral & - & & $33 / 222$ & 15 & \\
\hline Eye & - & & $27 / 222$ & 12 & \\
\hline Lung & - & & $7 / 222$ & 3 & \\
\hline Gastrointestinal & - & & $11 / 222$ & 5 & \\
\hline Liver & - & & $25 / 222$ & 11 & \\
\hline Joint/muscle & - & & $22 / 222$ & 10 & \\
\hline Genital & - & & $13 / 222$ & 6 & \\
\hline
\end{tabular}


Table 2 Continued

$A L L$ acute lymphoblastic leukemia, $A M L$ acute myeloid leukemia, $c G V H D$ chronic graft-versus-host disease, $C M L$ chronic myeloid leukemia, $H C T$ hematopoietic stem cell transplantation, IQR interquartile range

${ }^{a}$ Mann-Whitney $U$ test

${ }^{b}$ Fisher's exact test

Publisher's Note Springer Nature remains neutral with regard to jurisdictional claims in published maps and institutional affiliations. 